“(C) 2018 IEEE. Personal use of this material is permitted. Permission from IEEE must be obtained for all other uses, in any current or future media, including reprinting/republishing this material for advertising or promotional purposes, creating new collective works, for resale or redistribution to servers or lists, or reuse of any copyrighted component of this work in other works." 


\section{The Impact of Social versus Individual Learning for Agents' Risk Perception during Epidemics}

\author{
Shaheen A. \\ Abdulkareem \\ Centre of Studies of \\ Technology and Sustainability \\ Development (CSTM) \\ Faculty of Behavioural, \\ Management, and Social \\ sciences (BMS), University of \\ Twente \\ Enschede, The Netherlands \\ s.a.abdulkareem@utwente.nl
}

\author{
Ellen-Wien Augustijn \\ Department of Geo- \\ Information Process (GIP) \\ Faculty of Geo-Information \\ Science and Earth Observation \\ (ITC), University of Twente \\ Enschede, The Netherlands \\ p.w.m.augustijn@utwente.nl
}

\author{
Katarzyna Musial \\ Advanced Analytics Institute, \\ School of Software \\ Faculty of Engineering and IT, \\ University of Technology \\ Sydney, Australia \\ Katarzyna.Musial- \\ Gabrys@uts.edu.au
}

\author{
Yaseen T. Mustafa \\ Faculty of Science \\ University of Zakho (UoZ) \\ Kurdistan region, Iraq \\ Yaseen.Mustafa@uoz.edu.krd
}

\author{
Tatiana Filatova \\ School of Systems, \\ Management and Leadership \\ Faculty of Engineering and \\ Information Technology, \\ University of Technology \\ Sydney, Australia \\ tatiana.filatova@uts.edu.au
}

\begin{abstract}
Epidemics have always been a source of concern to people, both at the individual and government level. To fight outbreaks effectively, we need advanced tools that enable us to understand the factors that influence the spread of lifethreatening diseases.
\end{abstract}

Simulation tools are commonly used in assessing policy impacts in the health domain. Boulanger and Bréchet [1] in their evaluation of six paradigm models for policy-making recommended agent-based modelling (ABM) as the most promising modelling approach. ABMs provide a framework that allows representing an environment containing agents in a spatial and natural setting. They are based on a bottom-up structure to represent micro/macro relationships, accommodate agent heterogeneity and adaptive behaviour. ABMs also provide feedback between the spatial environment and cumulative agents' behaviour and can integrate a variety of data inputs such as aggregated, disaggregated and qualitative information or even commonsense knowledge [2][5].

As there is more and more data available in the area of disease spread, a new research direction has emerged - supporting $\mathrm{ABMs}$ with data-driven approached from machine learning (ML). ML can enhance the performance of ABMs, especially when the number of agents is large (e.g., pandemics) and the decision making of the agents is complex. In disease modelling, two elements are essential when using ML to enhance agent's behaviour: (i) risk perception, as this triggers the agents to change their coping behaviour and (ii) the factors that contribute to the risk perception (e.g., communication). The main idea behind using ML approaches in the context of $\mathrm{ABM}$ is to equip agents with the ability to learn by enabling them to optimise the decision-making process according to given criteria. ML techniques can provide agents with a more realistic way of learning and adjusting their behaviour [6].
Agents are social and can make individual decisions both or as part of a collective where they copy the decision from a group or group leader [7]. Agents might learn based on one of two principles: (i) isolated learning (centralised learning) or (ii) interactive learning (decentralised learning) [8]. In isolated learning, the agent learns independently without requiring any interaction with other agents. In interactive learning, several agents are engaged in the same process of learning, and they need to communicate and cooperate to learn effectively.

In this research, we use Bayesian Networks (BNs) to steer the behaviour of agents by representing risk perception and coping appraisal utilising a cholera model for Kumasi, a large city in Ghana [9]. Risk perception and coping appraisal are two stages of protection motivation theory that is presented by Roger (1983) [10]. We aim to evaluate the impact of interactive learning on the processes of risk perception and coping appraisal that will influence the spread of cholera. Two experiments are conducted in which we test isolated versus interactive learning in combination with including different types of communication. For the implementation, we use the BN model of Abdulkareem et al. (2018)[6].

The population of agents in the model is created using a synthetic population. We run each of the experiments 100 times. Every 10 runs a new synthetic population is generated. The experiments became stable after running them 50 times. Household agents who do not have access to tap water use BNs to perceive risk and make a protective decision about what to do in the face of epidemics. During the simulation, agents interact with their spatial environment and their neighbours. They gain information from the spatial environment (perceiving waste on the river or around river banks), from the media, and own prior knowledge of cholera cases. Moreover, household agents in the model may interact with neighbours. 


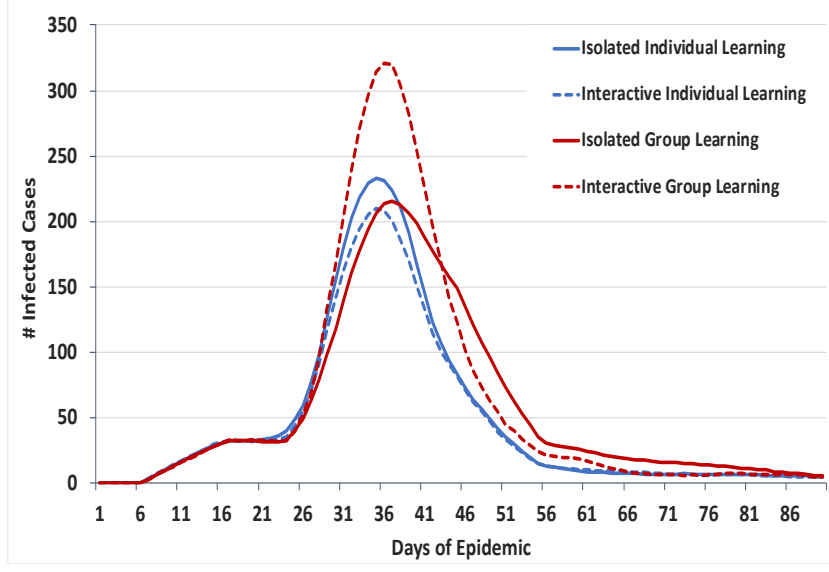

Figure 1: Epidemic curves of running models of the two experiments

Initial results indicate that isolated individual learning leads to less risk awareness and more infected cases compared to individual interactive learning (Figure 1). However, when comparing isolated group learning to interactive group learning, we found that groups with centralised learning lead to better decisions and lower peaks in the epidemic curves. When agents share their experience in their decentralised groups, this may result in making wrong decisions as the group members follow the majority in their groups. The sideeffect of engaging agents in decentralised groups is the dominance of the majority in their perception of the risk.

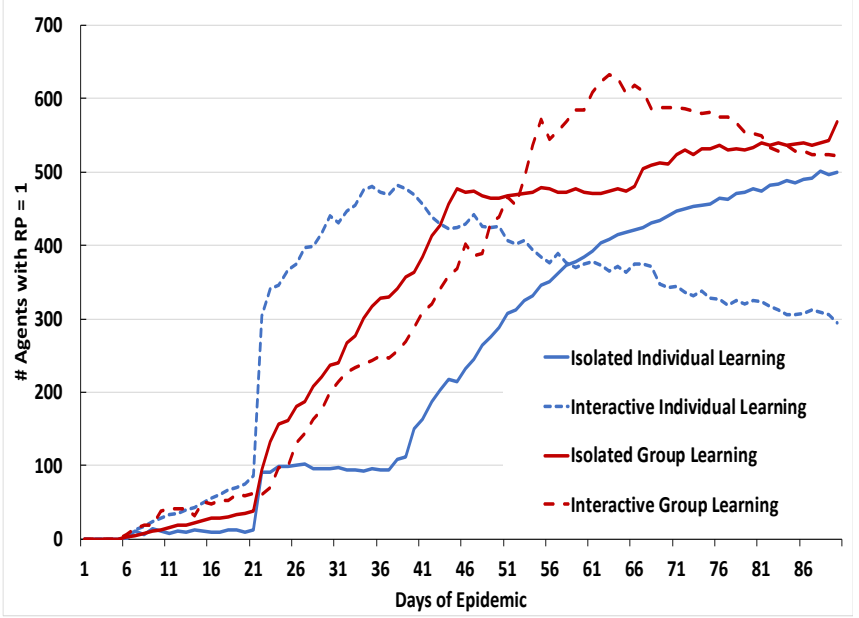

Figure 2: Development of agents' risk perception over time during of the experiments models

During the process of risk perception, agents with isolated individual learning show later responses compared to agents that learn interactively (Figure 2). Besides, when isolated agents start to perceive risk, they remain in that state even when the outbreak is over since they depend only on own knowledge and their experience with cholera disease. In isolated groups, agents who experienced cholera in their households will affect on the other group members and keep them aware of the cholera risk. This is why the risk perception of isolated groups remained increasing even though the epidemic reached its end.

The interactive learners, when the epidemic starts to fade out, resume their normal behaviour and lose their risk feeling. Only those agents who experienced cholera in their households they remained stimulate towards cholera risk.

Agents in interactive groups perceive risk later than individual interactive learners. This is because in decentralised groups, the majority of members are not aware of cholera risk and therefore they have a negative impact on the other members. However, when the number of infected cases increases this motivate the majority of the group members and they help their groups to perceive risk and make preventable decisions.

In this research, we investigated how the spread of epidemics depends on different techniques used for risk perception and decision making. We analysed how individual learning differs from collective learning and how it influences the dynamics of the epidemics. We concluded that social interactions of individuals have a direct impact on the dynamics of the outbreaks. This, in turn, can be used to promote certain behaviours to minimise the spread of epidemics proactively.

\section{REFERENCES}

[1] P.-M. Boulanger and T. Bréchet, "Models for policy-making in sustainable development: The state of the art and perspectives for research," Ecol. Econ., vol. 55, no. 3, pp. 337-350, Nov. 2005.

[2] L. An, "Modeling human decisions in coupled human and natural systems: Review of agent-based models," Ecol. Modell., vol. 229, pp. 25-36, Mar. 2012.

[3] T. Filatova, P. H. Verburg, D. C. Parker, and C. A. Stannard, "Spatial agent-based models for socio-ecological systems: Challenges and prospects," Environ. Model. Softw., vol. 45, pp. 1-7, Jul. 2013.

[4] S. de Marchi and S. E. Page, "Agent-Based Models," Annu. Rev. Polit. Sci., vol. 17, no. 1, pp. 1-20, May 2014.

[5] M. Fonoberova, V. A. Fonoberov, and I. Mezić, "Global sensitivity/uncertainty analysis for agent-based models," Reliab. Eng. Syst. Saf., vol. 118, pp. 8-17, Oct. 2013.

[6] S. A. Abdulkareem, E. W. Augustijn, Y. T. Mustafa, and T. Filatova, "Intelligent judgements over health risks in a spatial agent-based model," Int. J. Health Geogr., vol. 17, no. 1, pp. 1-19, Mar. 2018.

S. M. Carlson, R. E. White, and A. C. Davis-Unger, "Evidence for a relation between executive function and pretense representation in preschool children," Cogn. Dev., vol. 29, no. 1, pp. 1-16, 2014.

[8] S. Sen and G. Weiss, "Learning in multi-agent systems," in Multiagent Systems, G. Weiss, Ed. MIT Press, 1999, pp. 259-298.

[9] E. W. Augustijn, T. Doldersum, J. Useya, and D. Augustijn, "Agent-based modelling of cholera," Stoch. Environ. Res. Risk Assess., vol. 30, no. 8, pp. 2079-2095, 2016.

[10] R. W. Rogers, "Cognitive and Physiological Processes in Fear Appeals and Attitute Change: A Revised Theory of Porotection Motivation," in Social Psychophysiology: A Sourcebook, 1983, pp. 153-177. 